\title{
Editorial
}

\section{Recent Updates in Mammography}

\author{
Rasha M kamal \\ Professorof Rdiology, Women's imaging Unit, Cairo University
}

Corresponding Author: Rasha M kamal

\section{INTRODUCTION}

Two-dimensional (2D) mammography plays the most important role in all aspects of breast cancer detection, diagnosis and treatment ${ }^{1}$. It is the only screening modality proved to reduce mortality from breast cancer. However, the appearance of overlapping tissues on mammograms poses a significant obstacle to interpretation. When screening mammograms demonstrate a questionable finding, the results of follow-up diagnostic mammography and ultrasound (US), magnetic resonance (MR) imaging, or biopsy ultimately determine whether the finding is significant. The process causes anxiety for patients and incurs additional healthcare costs for findings that frequently are proved benign ${ }^{2,3}$.

Challenges of 2D mammography are structured noise which is created by the overlap of normal dense tissue structures within the breast, which are superimposed on each other in a standard two-dimensional mammogram. These overlapping structures can obscure a lesion, making it impossible for the radiologist to perceive it on a mammogram. This effect becomes more pronounced as the density of the breast increases. This may lead to reduction of the diagnostic sensitivity of mammography ${ }^{4}$. Breast tissue may also simulate the presence of a cancer that does not actually exist. This causes a loss of diagnostic specificity. Currently 2D mammography is the only x-ray imaging modality accepted for breast cancer screening, but for years researchers have tried to find improved technologies and new methods to supplement 2D mammography and provide better sensitivity and specificity ${ }^{1}$. It is this recognition that a significant percentage of breast cancers are not detected by screening mammography that has prompted recent interest in looking to other modalities as possible adjunctive screening tools ${ }^{4}$.

Film Screen Mammography and Digital Mammography

There are two main types of mammography: filmscreen mammography and digital mammography, also called full-field digital mammography or FFDM.
E-mail: rashaakamal@hotmail.com

It has been reported that $15-30 \%$ of detectable cancers in screening programs are not detected by screen-film mammography (SFM). In clinical series of patients, the sensitivity of SFM for breast cancer has been reported to be $80-90 \%$, but has been reported as low as $48 \%$ in extremely dense breasts. Factors implicated in missing cancers include the technique sensitivity, distracting lesions, the tumor growth rate, the tumor growth pattern and the background upon which the tumor is displayed. The introduction of digital mammography (DM) was expected to improve mammographic sensitivity for breast cancer detection. In randomized trials comparing SFM with DM, the sensitivity of mammography for cancer detection was increased in $\mathrm{DM}^{5}$.

High-quality full-field digital mammography has been available now for several years and is increasingly used for both diagnostic and screening mammography. In digital mammography, the screen film system is replaced by a detector which produces an electronic signal that is digitized and stored. A number of different detector technologies exist, which all have their specific advantages and disadvantages. The procedure and appearance of the machine and even the images produced are very similar to a traditional film mammogram. Full-field digital mammography (FFDM) has several potential benefits as compared with screen-film mammography (SFM) in mammography screening ${ }^{6}$. The advantage really comes from the ability to manipulate the image electronically. Because the digital machine is fast, patients spend less time in the exam room and rarely need to return for repeat images due to under or over exposures. Digital images can be manipulated for better views and they can be stored and retrieved more easily, and Brightness, darkness, or contrast can be adjusted and sections of an image can be magnified after the mammogram is complete making it easier to see subtle differences between tissues. The ability to increase contrast when imaging dense tissue is particularly important because dense breast tissue and malignant cells both appear to be white on a film mammogram. Digital images are easily stored and retrieved. Transmission of images from one physician to another is quick and easy. Diagnostic 
accuracy of digital mammography has been shown to be at least equivalent to film-screen mammography in a general screening population. Digital mammography is superior to screen-film mammography in younger women with dense breasts due to its ability to selectively optimize contrast in areas of dense parenchyma. This advantage is especially important in women with a genetic predisposition for breast cancer, where intensified early detection programs may have to start from 30 years of $\mathrm{age}^{7}$. Furthermore, digital technology provides a platform for new technologies, dedicated to advancing the early detection of breast cancer.

\section{COMPUTER AIDED DETECTION/ DIAGNOSIS (CAD):}

Computer-aided detection or computer-aided diagnosis (CAD), are procedures in medicine that assist doctors in the interpretation of medical images. CAD software algorithms analyze data from mammogram images to identify patterns associated with underlying breast cancers. After a radiologist completes an initial mammogram assessment, CAD marks potential abnormalities on the image for the consideration of the radiologist before making a final recommendation. In film mammography, CAD is coupled with a device to convert film mammograms to digital images and a viewing board while CAD is integrated directly into digital mammography environments ${ }^{8,9}$.CAD systems help scan digital images for typical appearances and to highlight conspicuous sections, such as possible diseases. CAD software analyzes the mammogram image and marks suspicious areas for radiologists to review, thus assisting them in determining which images could lead to invasive tumors. CAD was approved by the U.S. Food and Drug Administration in 1998 and has been incorporated into many mammography imaging practices, but its effect on the accuracy of interpretation has been unclear ${ }^{10}$. Computer aided detection systems for mammography have been available for a number of years ${ }^{11,12}$. They have been widely adopted in the US, where evidence that they can improve sensitivity has encouraged their use to improve cancer detection by a single reader. Butler et aL., found that CAD system correctly marked $87 \%(26 / 30)$ of those cancers that were clinically unsuspected (i.e., not at the location of the clinical finding $)^{13}$. In another study analysis of mammograms from more than 160,000 elderly women, use of CAD was associated with a slightly higher rate of breast cancer diagnosis. The increase was mainly because of more diagnoses of duct carcinoma in situ. The rate of invasive breast cancer overall was similar with and without CAD." CAD during screening mammography is associated with increased DCIS incidence, the diagnosis of invasive breast cancer at earlier stages, and increased diagnostic testing among women without breast cancer ${ }^{14,15}$.
Despite broad acceptance and use, it is unclear if the benefits of CAD during screening mammography outweigh its potential risks and costs. Ideally, CAD would lead to earlier detection of high-risk cancers, particularly invasive tumors, by improving sensitivity for these cancers and reducing the incidence of advanced stage breast cancer. However, the high sensitivity of CAD for mammographic calcifications may shift diagnostic attention to relatively indolent cancers, such as duct carcinoma in situ (DCIS), which often present with calcifications. In contrast, CAD is less likely to mark invasive cancers presenting as uncalcified masses and may therefore have little or no impact on the detection of higher-risk invasive breast cancer. Although meta-analyses suggest that CAD statistically significantly increases recall rates, clinical studies have generally included too few women with breast cancer to clarify whether CAD differentially affects detection of in situ vs invasive breast cancer, or whether CAD is associated with improved prognostic characteristics of breast cancer such as more localized stage or smaller tumor size. Such data are critical for understanding the potential of CAD to decrease breast cancer mortality - the ultimate goal of breast cancer screening ${ }^{15,16,17,18,19}$.

\section{D DIGITAL TOMOSYNTHESIS:}

Breast tomosynthesis technology is essentially a modification of a digital mammography unit to enable the acquisition of a three-dimensional (3D) volume of thinsection data. Images are reconstructed in conventional orientations by using reconstruction algorithms similar to those used in computed tomography $(\mathrm{CT})^{20,21}$. Units that are now in development for clinical use have dual functionality; that is, both two-dimensional (2D) digital mammography and breast tomosynthesis may be performed with the same unit. Breast tomosynthesis therefore has all the advantages of digital mammography, such as reproducibility, less image noise and fewer artifacts, consistent quality, and digital image processing ${ }^{22}$.

With the use of current breast tomosynthesis technology, the total radiation exposure to the patient from a two-view tomosynthesis acquisition is similar to, or less than that from conventional mammography. Breast tomosynthesis also has other exclusive advantages: Relative to conventional mammograms, the reconstructed tomosynthesis images provide improved visibility of objects within the selected cross section of breast tissue and, at the same time, reduced contrast and visibility of objects in overlying locations. Better delineation of the lesion border results in a more definitive interpretation ${ }^{21}$.

The goal of breast tomosynthesis is to make available a method for screening and diagnostic mammography which provides higher sensitivity and specificity than routine mammography. The analyses of breast tomosynthesis have shown the following clinical benefits: improvement of 
overall lesion detection and analysis, increased accuracy to either confirm or exclude a suspected abnormality and in particular detection capability of small breast cancers. The results indicate that breast tomosynthesis has the potential to significantly advance diagnostic mammography, as well as screening mammography in the future. Tomosynthesis studies have already shown a promise. Based on this clinical study, tomosynthesis of the breast will increase specificity. Studies also suggest that tomosynthesis might facilitate the detection of cancers at an earlier stage and a smaller size than is possible in 2D mammography ${ }^{23}$.

In cases with masses, the border of the mass, the number of masses (if multiple), and associated findings of dilated ducts or vessels and microcalcifications around the mass are better depicted on breast tomosynthesis images, especially in dense breasts (fig.1). The clearer depiction with tomosynthesis should allow easier differentiation between benign and malignant lesions. Therefore, the clinical application of breast tomosynthesis for screening should lead to a reduction in the recall rate, a higher positive predictive value for biopsy recommendation, and, eventually, a decrease in the number of unnecessary biopsies. The improvements in lesion perception and analysis also should lead to higher cancer detection rates (fig.2) ${ }^{20,21}$.

In a study performed by Ciatto, et al the introduction and routine use of tomosynthesis resulted in significant observed changes in recall rates from $8.7 \%$ to $5.5 \%$ $(P<0.001)$, nonsignificant changes in biopsy rates from 15.2 to 13.5 per 1000 screenings $(P=0.59)$, and cancer detection rates from 4.0 to 5.4 per 1000 screenings $(P=0.18)$. The invasive cancer detection rate increased from 2.8 to 4.3 per 1000 screening examinations $(P=0.07)$. The positive predictive value for recalls increased from $4.7 \%$ to $10.1 \%(P<0.001)^{24}$.
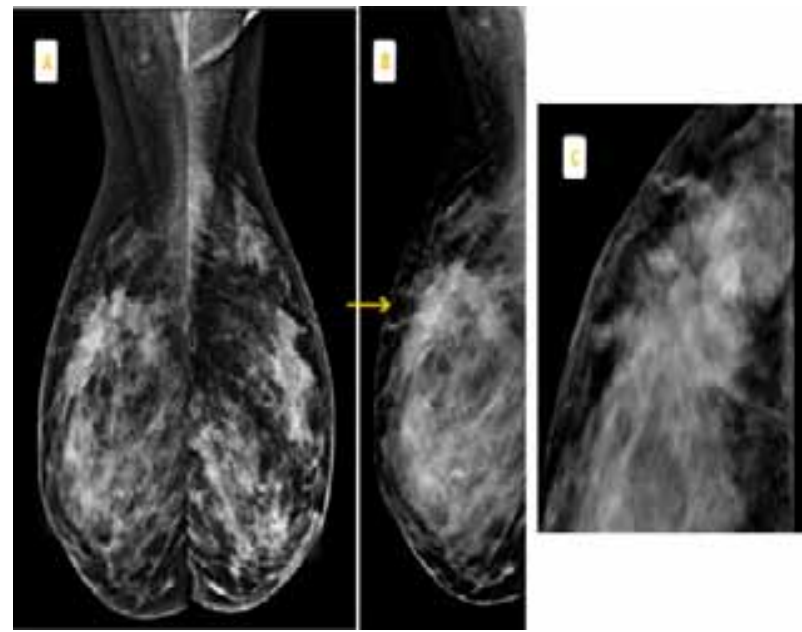

Fig. 1: [A] Heterogeneous dense parenchyma on 2 D mammography. [B] A spiculated outlined mass lesion was identified on Tomosynthesis slides; seen more evident on the magnification view [C].

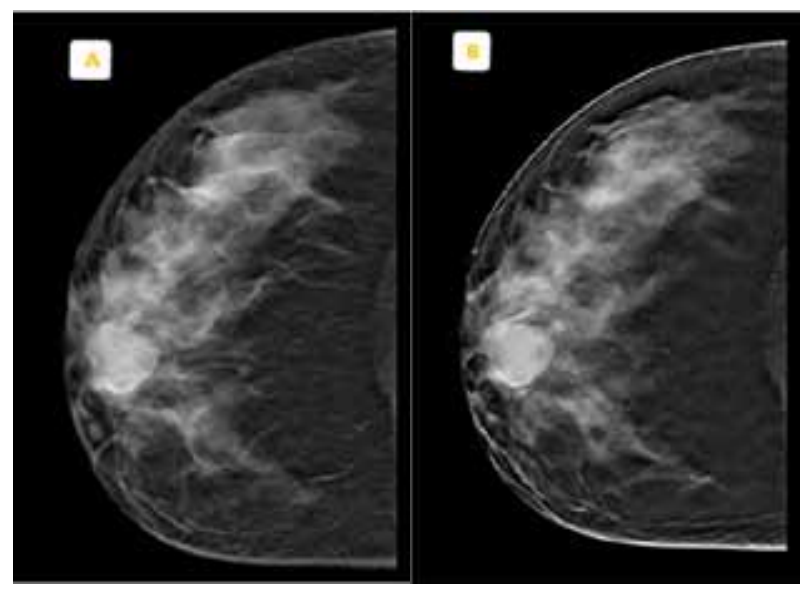

Fig. 2: A right retroareolar fibadenoma. On the FFDM images [A] it showed ill defined margins. It become more well defined on the Tomosynthesis images [B].

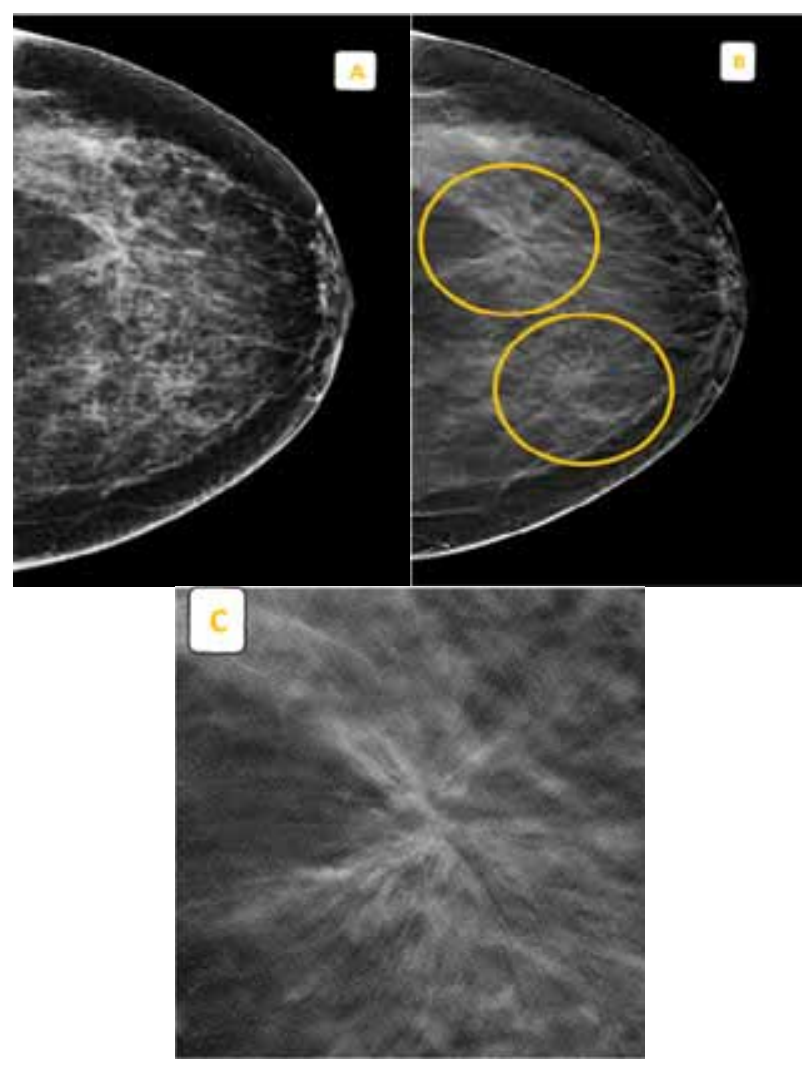

Fig. 3: Tiny spiculated mass lesions that were not identified on the FFDM [A]. Multicentric carcinomas were identified on the Tomosynthesis slides [B]; being more evident on the magnified view.

\section{CONTRAST ENHANCED SPECTRAL MAMMOGRAPHY:}

Despite advances in resolution and contrast, mammography remains a diagnostic imaging modality where image interpretation is very difficult. Breast radiographs are generally examined for the presence of malignant masses and indirect signs of malignancy such as 
the presence of micro-calcifications and skin thickening. Unfortunately, it is unlikely that major improvements in imaging performance will be achieved by technical advances in screen/film mammography alone. The major reason for poor visualization of some malignant masses is the minor difference in x-ray attenuation between normal glandular tissues and malignant disease. This fact makes the detection of small malignancies problematic, especially in younger women who have denser breast tissue where surrounding fibroglandular tissue decreases the conspicuity of lesions ${ }^{25}$. Even when tumors are detected, the full extent of disease may not be clearly depicted. Because cancers and fibroglandular tissue show similar x-ray absorption, tumor enhancement with a contrast medium should improve cancer detection ${ }^{26}$.

Many methods for imaging angiogenesis in vivo have been developed in the last few years. Injection of iodinated contrast medium is one of these clinical applications enabled by full-field digital mammography technology. The growth and metastatic potential of tumors can be directly linked to angiogenesis. Growth beyond a few millimeters in diameter requires the formation of new blood vessels to supply the oxygen and nutrients necessary for survival .Tumor angiogenesis factors stimulate formation of abnormal vessels that leak and shunt blood. Therefore, imaging methods with contrast medium potentially can aid in the detection and diagnosis of cancer ${ }^{26}$.

Two contrast enhanced digital mammography (CEDM) techniques are under development, the dual energy technique and the temporal technique. The dual energy technique exploits the energy dependence of the X-ray attenuation through materials of different composition in the breast, specifically iodine and soft tissues. A pair of low and high-energy images is obtained after the administration of an iodinated contrast medium agent. Then, the two images are combined to enhance contrast uptake $\operatorname{areas}^{27}$. The high-energy exposures require an adaptation of the digital mammography system. The X-ray spectrum must be shaped so that the X-rays have energies above the $\mathrm{K}$-edge of iodine $(33.2 \mathrm{keV})$. First an iodinated contrast agent is injected preferably using a power injector at a high flow rate. Then the breast is compressed and a pair of low and high-energy images is acquired. Image analysis requires the combination of low-energy and high-energy images to generate a dual energy image with contrast uptake information. The resulting images are reviewed using reading criteria based on contrast enhancement intensity and morphology. The duration of examination ranges from 5 to $10 \mathrm{~min}$ depending on the number of projections. The total X-ray dose delivered to the patient for a pair of low and high-energy images is estimated to be 20 $50 \%$ higher than the dose needed for one projection in conventional mammography, depending on the breast thickness and tissue composition ${ }^{28}$.

The second technique is the temporal subtraction technique The temporal subtraction technique produces high-energy digital mammography images before and after contrast medium injection. To enhance visualization of contrast medium in lesions, the pre-contrast image is subtracted from the post-contrast images. Similar to the dual energy technique it is necessary to adapt the digital mammography system such that the sensitivity of the imaging technique to low concentrations of iodine is maximized. Voltages ranging between 45 and $49 \mathrm{kVp}$ are typically used instead of $26-32 \mathrm{kVp}$ for conventional digital mammography. The patient has to be comfortably settled in order to avoid motions during the acquisition of the image sequence. A light breast compression is used for all mammography images, strong enough to limit motion but limited to avoid reducing the blood flow. All images are acquired within a single breast compression ${ }^{28}$.

Each technique has some advantages and drawbacks The temporal subtraction approach offers the possibility to analyze the enhancement and washout patterns of the contrast material into and out of the lesion. The temporal subtraction CEDM enhancement curve is similar to that of enhancement curves obtained with gadolinium contrast examinations with MRI which have been found to be useful to assess the probability of malignancy. However, one of the problems with the temporal approach is that to get artifact-free subtracted images, the pre and post-contrast images must register with a high accuracy. Unfortunately, it is difficult to keep the breast motionless during several minutes, especially when a light compression is applied. Dual energy CEDM does not provide information about the kinetics of tumor enhancement because the number of images performed after contrast medium injection is limited to control the level of radiation exposure. However, dual energy technique allows the acquisition of multiple views of the same breast (cranio-caudal and medio-lateral oblique projections) or bilateral examination. Moreover, it is less sensitive to patient motion than dynamic CEDM because the time between low and high-energy exposures is very short. In addition, because the breast compression duration is reduced, dual energy CEDM is better tolerated by the patient than temporal CEDM. Finally, the radiation exposure is higher with dual energy $\mathrm{CEDM}^{28}$.

Initial clinical experience has shown the ability of CEDM to map the distribution of neo-vasculature induced by cancer at the high resolutions possible with digital mammography. CEDM should result in a simple way to enhance the detection of some breast cancers, to improve the characterization of breast lesion and to provide some prognostic factors of breast carcinoma. 
However, positive and negative predictive values should be prospectively evaluated on extensive clinical experience and in selected patient population. Contrastenhanced digital mammography potentially may be useful in the identification of lesions in the mammography dense breast. As in MR imaging, other applications may be in the identification of the extent of disease or in the detection of an otherwise occult carcinoma that has manifested with axillary metastases. This information may aid in the diagnosis and guidance of core-needle biopsy or excision of these lesions. Furthermore, with the increasing availability of digital mammography, contrastenhanced digital mammography will become accessible and relatively inexpensive compared with current MR imaging technology. Its place among the other breast imaging methods is still to be specified and comparison with breast MRI should be described. Moreover, future technical improvement can be expected to improve contrast visualization with lower radiation dose allowing more views and different image timing. CEDM will probably benefit of other digital mammography improvements such as tomosynthesis ${ }^{27,28}$.

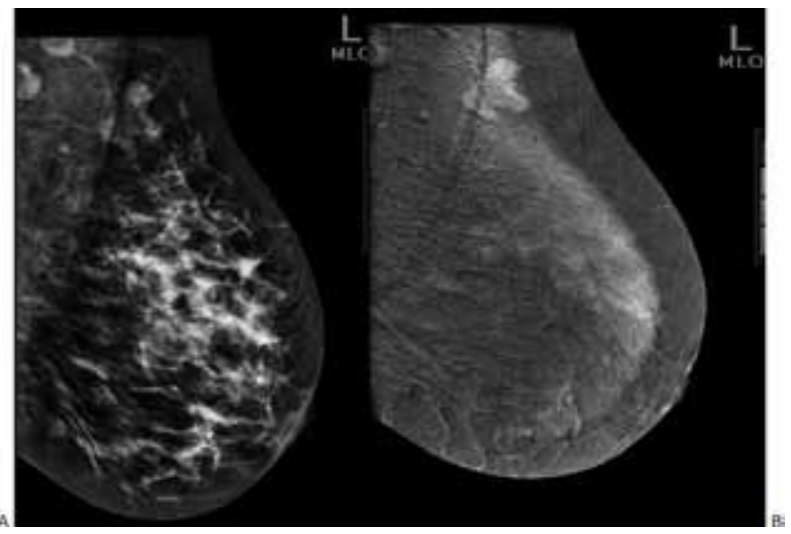

Fig. 4: Heterogeneously dense parenchyma of the left breast shows left axillary tail focal asymmetry on the FFDM films [A]. The mass is better depicted and better defined on the contrast images [B].

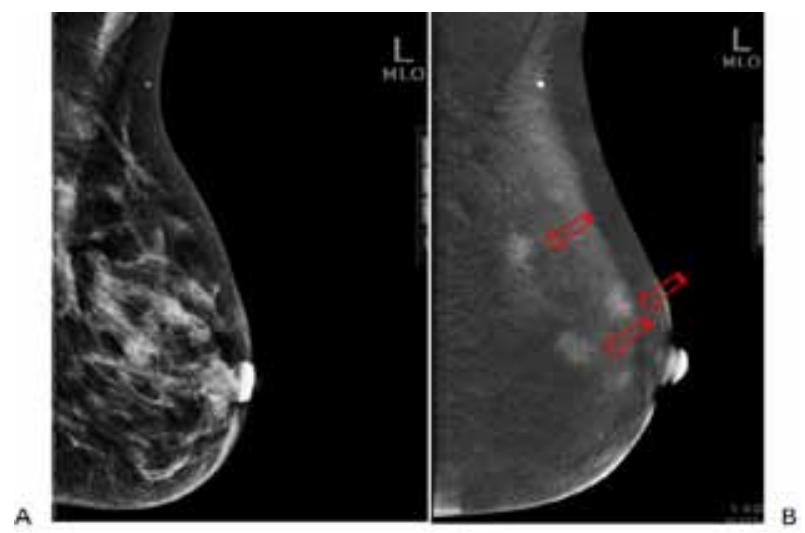

Fig. 5: Heterogeneous dense parenchyma of the left breast with no identified abnormalities on FFDM film (A). Underlying multi focal malignant mass lesions are seen on CEDM film (B).

\section{CONCLUSION}

In this article, we reviewed the current state of the art in technology for digital mammography. In general, the goal of mammography is the detection, characterization, and evaluation of findings suggestive of breast cancer. It is essential that all mammography be performed and interpreted with the highest quality possible. Digital mammography can assist the introduction of new applications, with the potential of improving the diagnostic and detection capabilities of mammography and thus overcoming its limitations.

\section{REFERENCES}

1. Mari V. Three-Dimensional (3D) Digital Breast Tomosynthesis (DBT) in the Early Diagnosis and Detection of Breast Cancer. Tampereen teknillinen yliopisto. Julkaisu - Tampere University of Technology. Publication, 2008; 594, http://URN.fi/URN:NBN:fi:tty-200810021130.

2. Park JM, Franken EA, Garg M, Fajardo LL, Niklason LT. Breast Tomosynthesis: Present Considerations and Future applications. RadioGraphics 2007, 27: 231-40.

3. LewinJM, D'Orsi CJ, Hendrick RE. Digital mammography. Radiol Clin North Am2004; 42(5): 871-884, vi.

4. Rafferty E. Tomosynthesis: New Weapon in Breast Cancer Fight. Imaging Economics, 2004.

5. Anderson J. Ikeda DM, Zackrisson S, Ruschin M, Svahn T, Timberg T. Breast tomosynthesis and digital mammography: a comparison of breast cancer visibility and BIRADS classification in a population of cancers with subtle mammographic findings. Eur Radiol, 2008, 18: 2817-25.

6. Skaane P. Studies comparing screen-film mammography and full-field digital mammography in breast cancer screening: updated review. Acta Radiol. 2009 Jan;50(1):3-14.

7. Bick U, Diekmann F. Digital mammography: what do we and what don't we know? Eur Radiol. 2007 Aug;17(8):1931-42.

8. Fenton JJ, Abraham L, taplin SH, Geler BM, Carnev PA, Dorsi C, Elmore JG, and Barlow WE. Effectiveness of Computer-Aided Detection in Community Mammography Practice. JNCI J Natl Cancer Inst (2011): 1093.

9. Rao VM, Levin DC, Parker L, Cavanaugh B, Frangos AJ, Sunshine JH. How widely is computer-aided detection used in screening and diagnostic mammography? J Am Coll Radiol. 2010;7(10):802-805.

10. Fenton JJ, Taplin SH, et al. Influence of Computer-Aided Detection on Performance of Screening Mammography. NEJM, Vol. 356, No. 14. April 5, 2007.

11. Taylor P, Potts HW. Computer aids and human second reading as interventions in screening mammography: two systematic reviews to compare effects on cancer detection and recall rate. Eur J Cancer, 2008, 44:798-807.

12. Gilbert FJ, Astley SM, Gillan MG et al. Single reading with computer-aided detection for screening mammography. N Engl J Med, 2008, 359:1675-1684. 
13. Butler SA, Gabbay RJ, Kass DA, Siedler DE, O'shaughnessy KF, Castellino RA. Computer-aided detection in diagnostic mammography: detection of clinically unsuspected cancers. AJR Am J Roentgenol. 2004 Nov;183(5):1511-5.

14. McReady N. Mammography With Computer-Aided Detection: A Mixed Blessing? Medscape Medical News 2013;(15)

15. Fenton JJ, Xing G, Elmore JG, Bang H, Chen SL, Linfors KK and Baldwin LM. Short-Term Outcomes of Screening Mammography Using Computer-Aided Detection: A Population-Based Study of Medicare Enrollees. Ann Intern Med. 2013;158(8):580-587.

16. Birdwell RL. The preponderance of evidence supports computer-aided detection for screening mammography. Radiology, 2009; 253(1):9-16.

17. Philpotts L. Can computer-aided detection be detrimental to mammographic interpretation? Radiology, 2009; 253(1):17-22.

18. Autier P, Hery C, Haukka J, Boniol M, Byrnes G. Advanced breast cancer and breast cancer mortality in randomized controlled trials on mammography screening. J Clin Oncol, 2009;27(35): 5919-5923.

19. Brem RF, Rapelyea JA, Zisman G, Hoffmeister JW, Desimio MP. Evaluation of breast cancer with a computeraided detection system by mammographic appearance and histopathology. Cancer, 2005;104(5): 931-935.

20. Park JM, Frankin EA, Fajrdo LL, Niklason LT. Breast Tomosynthesis: Present Considerations and Future Applications. RadioGraphics, 27, S231-S240.
21. SmithA. Full-field breast tomosynthesis. Radiol Manage2005; 27(5): 25-31.

22. NiklasonLT, Kopans DB, Hamberg LM. Digital breast imaging: tomosynthesis and digital subtraction mammography. Breast Dis1998; 10(3-4): 151-164

23. Mari V Three-Dimensional (3D) Digital Breast Tomosynthesis (DBT) in the Early Diagnosis and Detection of Breast Cancer Tampereen teknillinen yliopisto. JulkaisTampere University of Technology. Publication; 594, 2006. http://URN.fi/URN:NBN:fi:tty-200810021130.

24. Ciatto S, Houssami N, Bernardi D, Caumo F, Pellegrini M, Brunelli S, Tuttobene P, Bricolo P, Fantò C, Valentini $\mathrm{M}$, Montemezzi S, Macaskill P. Integration of 3D digital mammography with tomosynthesis for population breastcancer screening (STORM): a prospective comparison study. Lancet Oncol. 2013 Jun; 14(7):583-9.

25. Johns PC and Yale MJ. X-ray characterization of normal and neoplastic breast tissues. Physics in Medicine and Biology, Vol. 32, No. 6, pp. 675-695, Feb. 1987.

26. Jong RA, Yaffe MJ, Skarpathiotakis M, Shumak RS, Danjoux NM, Gunesekara A, Plewes DB. Contrastenhanced Digital Mammography: Initial Clinical Experience. Radiology 2003, 228:842-850.

27. Puong S, Bouchevreau X, Patoureaux F, Iordache R, Muller S. Dual-energy contrast-enhanced digital mammography using a new approach for breast tissue cancelling. In: Proceeding of SPIE, Medical Imaging. 2007.

28. Dromaina C, balleyguiera C, Aldera G, Garbayb SD. Contrast-enhanced digital mammography. European Journal of Radiology 69 (2009) 34-42. 